\title{
NEURO-FUZZY MODELING OF A SPONGE-TYPE MR DAMPER
}

\author{
Manuel T. Braz-César ${ }^{1}$, Rui C. Barros ${ }^{2}$ \\ ${ }^{1}$ Polytechnic Institute of Bragança \\ Campus de Santa Aplónia - Apt. 134 - 5301-857 Bragança - Portugal \\ e-mail: brazcesar@ipb.pt \\ ${ }^{2}$ Faculty of Engineering of the University of Porto \\ Rua Dr. Roberto Frias, s/n 4200-465 Porto - Portugal \\ rcb@fe.up.pt
}

Keywords: MR damper, ANFIS, Fuzzy model.

\begin{abstract}
Numerical modeling of MR dampers based on parametric models constitutes one of the main methodologies to simulate the behavior of this type of devices. However, its highly non-linear nature and also its inherent rheological behavior make this type of numerical modeling harsh and complicated, which hinders the development of simple models capable to cover all aspects associated with the proper numerical simulation of the damper behavior and therefore usually complex parametric models involving several parameters are required to achieve a reliable and accurate representation of its rheological behavior. Hence, nonparametric models represent another feasible approach to simulate the complex non-linear behavior of MR dampers although in this case allowing to obtain a wide-ranging numerical model without the need to define or identify a large number of model parameters. In this context, we attempt to model and predict the response of a sponge-type MR damper using a nonparametric modeling technique based on an Adaptive Neuro-Fuzzy Inference System (ANFIS) model. Initially, the basic structure of this data modeling technique is presented and the main aspects regarding the development of a neuro-fuzzy model for MR dampers are addressed. Then, an ANFIS modeling technique is developed to obtain a non-parametric model for the $M R$ damper. Finally, a comparison between the numerical and experimental results will be presented to validate the selected modeling technique.
\end{abstract}




\section{INTRODUCTION}

Non-parametric models represent an alternative methodology to numerically simulate the complex behaviour of active and semi-active devices. Among these modelling techniques, neuro-fuzzy models incorporate pattern recognition capabilities of neural networks with the non-linear mapping modelling of fuzzy logic systems which make them a particularly suitable non-parametric modelling approach to characterize the highly non-linear behaviour of MR dampers. A generic schematic representation of a neuro-fuzzy model is shown in Figure 1.

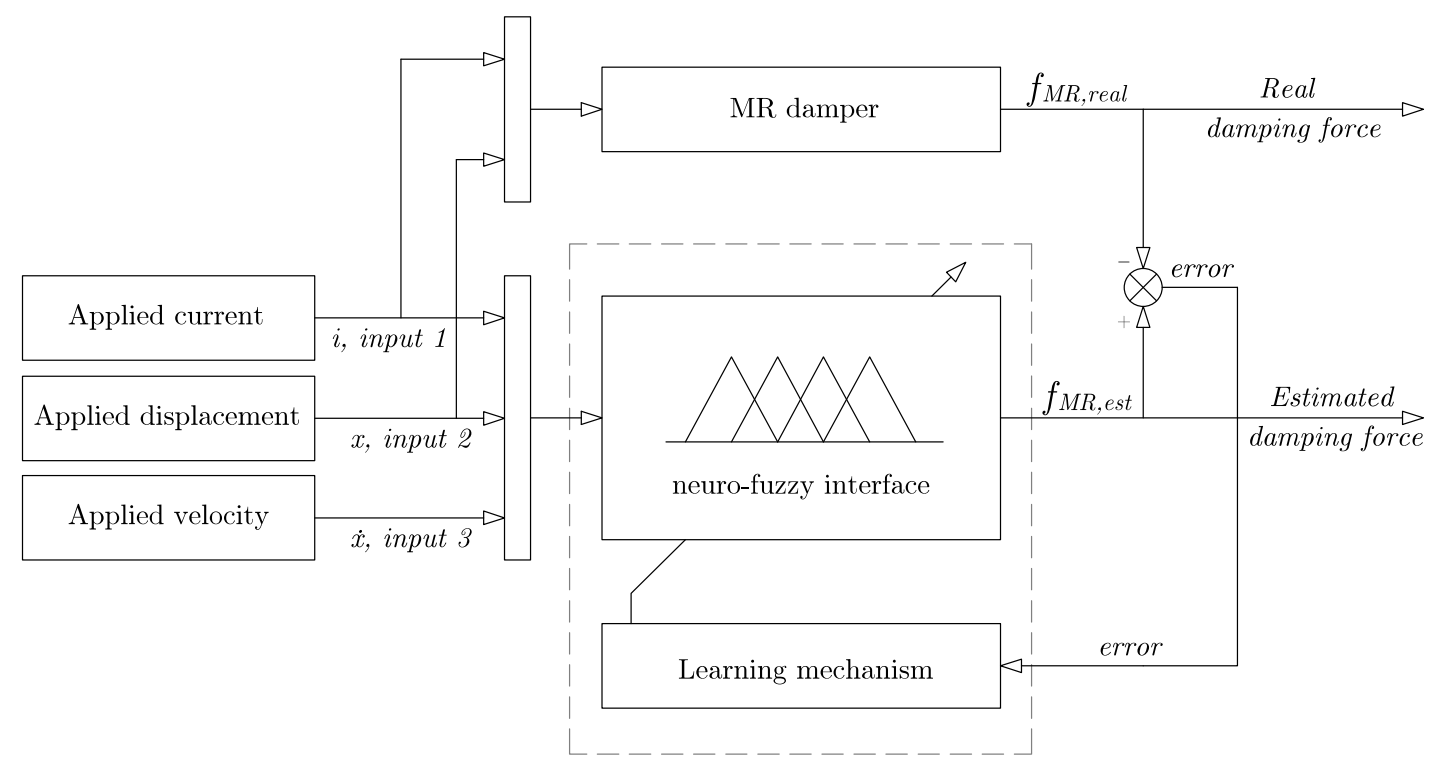

Figure 1: Neuro-fuzzy modeling of a MR damper.

Adaptive Neuro-Fuzzy Inference System (ANFIS) algorithm presents the advantage of providing automatic tuning of a Sugeno-type fuzzy inference system (FIS) to model the relationships of the input variables and their important parameters such as the mechanical excitation and operating current to generate a single output. Moreover, numerical simulation based on fuzzy logic represents a robust and reliable modelling methodology that allows the numerical model to account for some uncertainties associated with the typical hysteretic behaviour of MR devices but, especially due to its ability to represent unpredicted responses that are usually not quantified or considered with more accurate models (e.g., parametric models), since fuzzy logic models use continuous membership functions to relate inputs and outputs. Although FIS models can be considered less accurate than other numerical models, they have been shown to correctly represent the experimental response of MR dampers, presenting a good compromise between computational time and numerical precision [1-6].

Hence, the present paper explores the use of this neuro-fuzzy technique in modelling the response of a sponge-type MR damper. The application of an ANFIS algorithm to emulate the non-linear behaviour of MR dampers device will be investigated to demonstrate the capabilities of this hybrid intelligent system as a feasible modelling approach to map the relationships between inputs and outputs in order to create a fuzzy model for these devices even with uncertain or imprecise knowledge about its behaviour. The proposed modelling approach can be applied to estimate the non-linear hysteretic response of this type of devices between different operating currents and mechanical excitations and is validated over a set of experimental and numerical datasets of the sponge-type MR damper. 


\section{ADAPTIVE NEURO-FUZZY INFERENCE SYSTEM (ANFIS)}

Neuro-adaptive learning techniques represent a simple methodology for the fuzzy modeling procedure to learn information about a dataset in order to compute the membership function parameters that best allow the associated fuzzy inference system to track a given input/output data. ANFIS uses a hybrid learning algorithm that combines the backpropagation gradient descent and least squares methods to create a fuzzy inference system whose membership functions are iteratively adjusted according to a given set of input and output data $[3,4]$. The reasoning scheme of ANFIS architecture is shown in Figure 2.

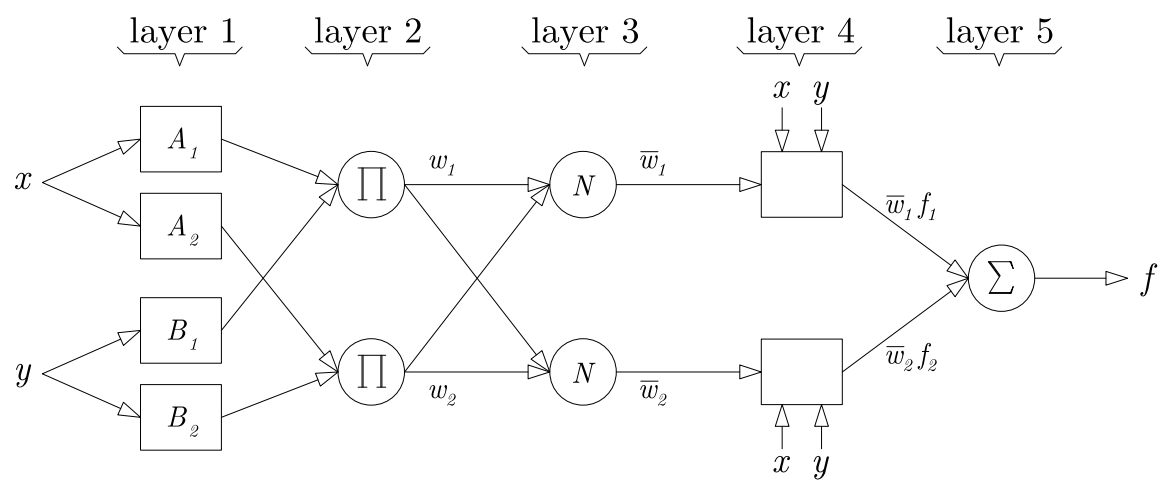

Figure 2: Adaptive Neuro-Fuzzy Inference System or ANFIS.

Soft computing methods represent a relatively recent modeling technique of MR dampers that have been shown to be effective in representing the non-linear response of these devices. The development of a neuro-fuzzy model of a MR damper involve four main steps:

1) Definition of input variables and the corresponding FIS membership functions (the FIS output is the desired damper force);

2) Selection of experimental or artificial data sets to generate training and checking data;

3) Use of ANFIS optimization algorithm for training the FIS membership function parameters to model the set of input/output data by mapping the relationship between inputs and outputs in order to generate a fuzzy model of the MR damper;

4) Validation of the resulting fuzzy model.

ANFIS system training procedure is summarized in the flowchart shown in Figure 3. The process begins by obtaining a training data set and checking data sets. The training data is used to find the premise parameters for the membership functions (MFs are dependent on the system designer). A threshold value for the error between the actual and desired output is determined. The consequent parameters are found using the least-squares method. If this error is larger than the threshold value, then the premise parameters are updated using the gradient decent method. The process end when the error becomes less than the threshold value. Checking data set can then be used to compare the model with the actual system. 


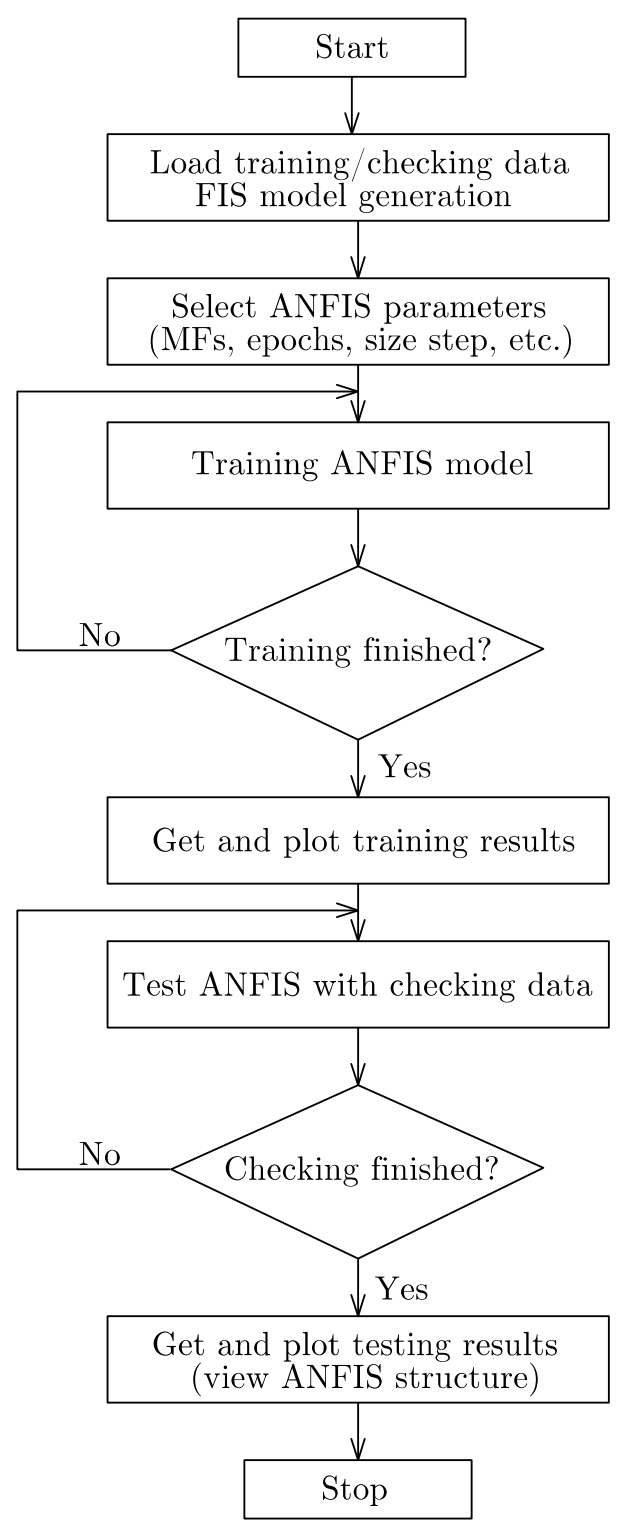

Figure 3: Flowchart of ANFIS training.

After the ANFIS training process is complete, a fuzzy model of the MR damper is obtained based on the training data, training options and type/number of membership functions previously defined by the system designer.

It is important to notice that the fuzzy inference system and the corresponding input (displacement, velocity and operating current) and output (damper force) membership functions produced by the ANFIS training algorithm must be capable to conveniently represent the actual behavior of the device. Although the training data may have significant information about the behavior of the MR damper, the resulting fuzzy model may not accurately represent the damper response (incorrect training data, some wrong or uneven data points, singularities in the device response, etc.). Thus, a final verification is required to ensure realistic results of the fuzzy model, which can be done by performing an assessment of the fuzzy surfaces to confirm realistic input/output magnitudes and geometry. 


\section{FUZZY MODELING WITH ANFIS}

MR dampers are semi-active devices whose damping characteristics can be modified in real time due to the capability to adjust the resistance to flow of a MR fluid within the damper through the application of a magnetic field. The particular properties of the MR fluid allow variations in the damping force that can be controlled by varying an applied current. Thus, the hysteretic behaviour of a MR damper is current dependent but also function of the amplitude of the excitation [9-11].

The present section describes the application of ANFIS to develop a neuro-fuzzy model for a sponge-type MR damper (RD-1097-1 model). It is intended to optimize a fuzzy inference system by training a family of membership functions according to a predetermined input and output data set related with the damper behaviour. Thus, the piston displacement and velocity, and the operating current represent the three fuzzy variables assigned to the input membership functions and the damper force represents the fuzzy output variable. Generalized bell-shaped membership functions are used to represent the input variables. The fuzzy parameters assigned to each fuzzy input variable, i.e., the properties of the selected MFs (number, type and universe of discourse) are summarized in Table 1.

\begin{tabular}{c|c|c|c|c}
\hline \multirow{2}{*}{ Data } & Fuzzy variable & Type & $\begin{array}{c}\text { Number } \\
\text { of MFs }\end{array}$ & Universe of discourse* $^{* *}$ \\
\hline \multirow{2}{*}{ Input MFs } & - Piston displacement & $\begin{array}{c}\text { Generalized } \\
\text { bell-shaped }\end{array}$ & 2 & $\left|-\mathrm{x}_{\max }, \mathrm{x}_{\max }\right|$ with $\mathrm{x}_{\max }=20 \mathrm{~mm}$ \\
\cline { 2 - 5 } & - Piston velocity & $\begin{array}{c}\text { Generalized } \\
\text { bell-shaped }\end{array}$ & 3 & $\left|-\dot{x}_{\max }, \dot{x}_{\max }\right|$ with $\dot{x}_{\max }=14 \mathrm{~cm} / \mathrm{s}$ \\
\cline { 2 - 5 } & - Operating current & $\begin{array}{l}\text { Generalized } \\
\text { bell-shaped }\end{array}$ & 2 & $\left|0, I_{\max }\right|$ with $\mathrm{I}_{\max }=0.50 \mathrm{~A}$ \\
\hline
\end{tabular}

Table 1: Fuzzy MFs parameters (RD-1097-1 MR damper).

According with the device specifications the sponge-type MR damper operates with an operating currents up to 1.0 A (intermittent operation). The maximum stroke is around $\pm 25 \mathrm{~mm}$ within a damping force range of $9 \mathrm{~N}(0.0 \mathrm{~A})$ and $100 \mathrm{~N}(1.0 \mathrm{~A})$. The expected operational ranges for this MR damper are $\mathrm{x}= \pm 20 \mathrm{~mm}, \dot{\mathrm{x}}= \pm 14.0 \mathrm{~cm} / \mathrm{s}$ and $\mathrm{I}=0.5 \mathrm{~A}$. Based on the experimental results, the maximum damping force is around $30 \mathrm{~N}$. These values represent the upper and lower boundaries of the proposed neuro-fuzzy model (i.e., $\mathrm{f}_{\min }=2 \mathrm{~N}, \mathrm{f}_{\max }=30 \mathrm{~N}, \mathrm{x}_{\max }=20$ $\mathrm{mm}, \dot{\mathrm{x}}_{\max }=14.0 \mathrm{~cm} / \mathrm{s}, \mathrm{I}_{\min }=0.0 \mathrm{~A}$ and $\mathrm{I}_{\max }=0.5 \mathrm{~A}$ ). Hence, the training data should include enough information to cover the entire spectrum of operation of the MR damper within these ranges of values since the precision of the fuzzy model is mainly dependent on the training data used for the FIS optimization with ANFIS.

This type of MR devices are usually commanded through a voltage-to-current controller unit designated as Wonder Box Device Controller and sold as a complementary product for MR devices. The device provides a closed-loop current control to compensate for changing electrical loads up to the limits of the power supply and can be used to investigate the control possibilities of the LORD MR technology since it can be operated as an interface device for PLC or computer control of the MR fluid devices. This controller was connected to a voltage power supply unit in order to feed the MR damper with a constant voltage converted to current supply through the Wonder Box. 
In this case, a set of numerical signals based on experimental results was used to develop the neuro-fuzzy model. More specifically, the numerical based fuzzy model was developed from the numerical results of the Bouc-Wen model which in turn was developed from experimental data obtained from several experimental tests. Thus fuzzy model was developed to ensure good performance in solving the fuzzy parameter prediction problem by using artificial data inputs to generate the training data set. The aim is to verify the efficiency of the optimization algorithm to predict the damper response when a smooth, regular and plentiful data set is available for the training procedure. The artificial data set used for training the FIS of the neuro-fuzzy model for the sponge-type MR damper is illustrated in Figure 4.
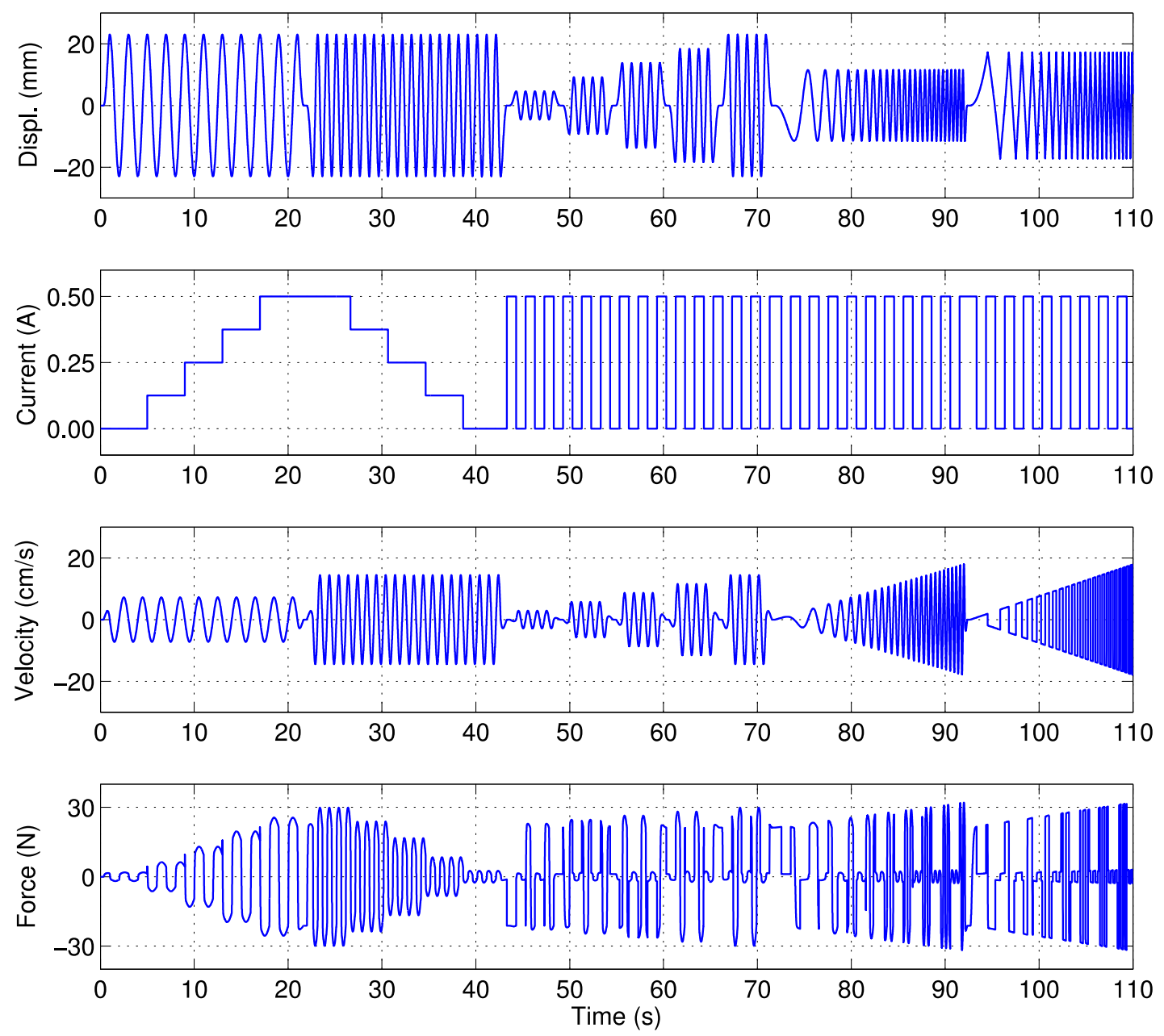

Figure 4: Training data for fuzzy model A (RD-1097-1 MR damper).

Thus, a series of training sequences comprising five types of artificial displacement sequence inputs were used to generate the data set for training the fuzzy inference system of the proposed numerical based fuzzy model. Constant displacement (CD), triangular wave (TW), amplitude-modulated (AM) and frequency-modulated (FM) are the displacement sequences used to represent the MR damper dynamics and the hysteresis behavior under changes in the magnitude and frequency of excitation for stepped increments of the operating current level. To improve ANFIS optimization, displacements outside the expected operating range (i.e., $x=$ $\pm 20 \mathrm{~mm}$ ) were trimmed. The main characteristics of the five displacement sequences are presented in Table 2. 
The signal sequences have 110000 data points. A training data set containing a total of 22000 data points $(110 \mathrm{sec}$.) was generated using a time step of $0.005 \mathrm{sec}$. In this case, odd numbered values were used for training and even numbered values for checking (50\%-50\% alternating training-checking partition).

\begin{tabular}{c|c|c|c|c}
\hline Case & Type of displacement & $\begin{array}{c}\text { Amplitude } \\
(\mathbf{m m})\end{array}$ & $\begin{array}{c}\text { Frequency } \\
(\mathbf{H z})\end{array}$ & $\begin{array}{c}\text { Current } \\
(\mathbf{A})\end{array}$ \\
\hline $1 / 2$ & Constant Displacement (CD) & $20^{*}$ & $0.5 / 1.0$ & $0.0-0.5(5$ steps) \\
\hline 3 & Amplitude-Modulated (AM) & $4-20^{*}(5$ steps $)$ & 3.0 & $0.0-0.5(5$ steps) \\
\hline 4 & Frequency-Modulated (FM) & 12 & $0.5-3.0$ & 0.0 and 0.5 \\
\hline 5 & Triangular Wave (TW) & 18 & $0.5-3.0$ & 0.0 and 0.5 \\
\hline
\end{tabular}

Table 2: Artificial training data (RD-1097-1 MR damper).

The membership function parameter adjustment was performed using the ANFIS training routine included in the MATLAB fuzzy logic toolbox [12]. The input membership functions for command voltage, displacement and velocity before and after training are shown in Figure 5.
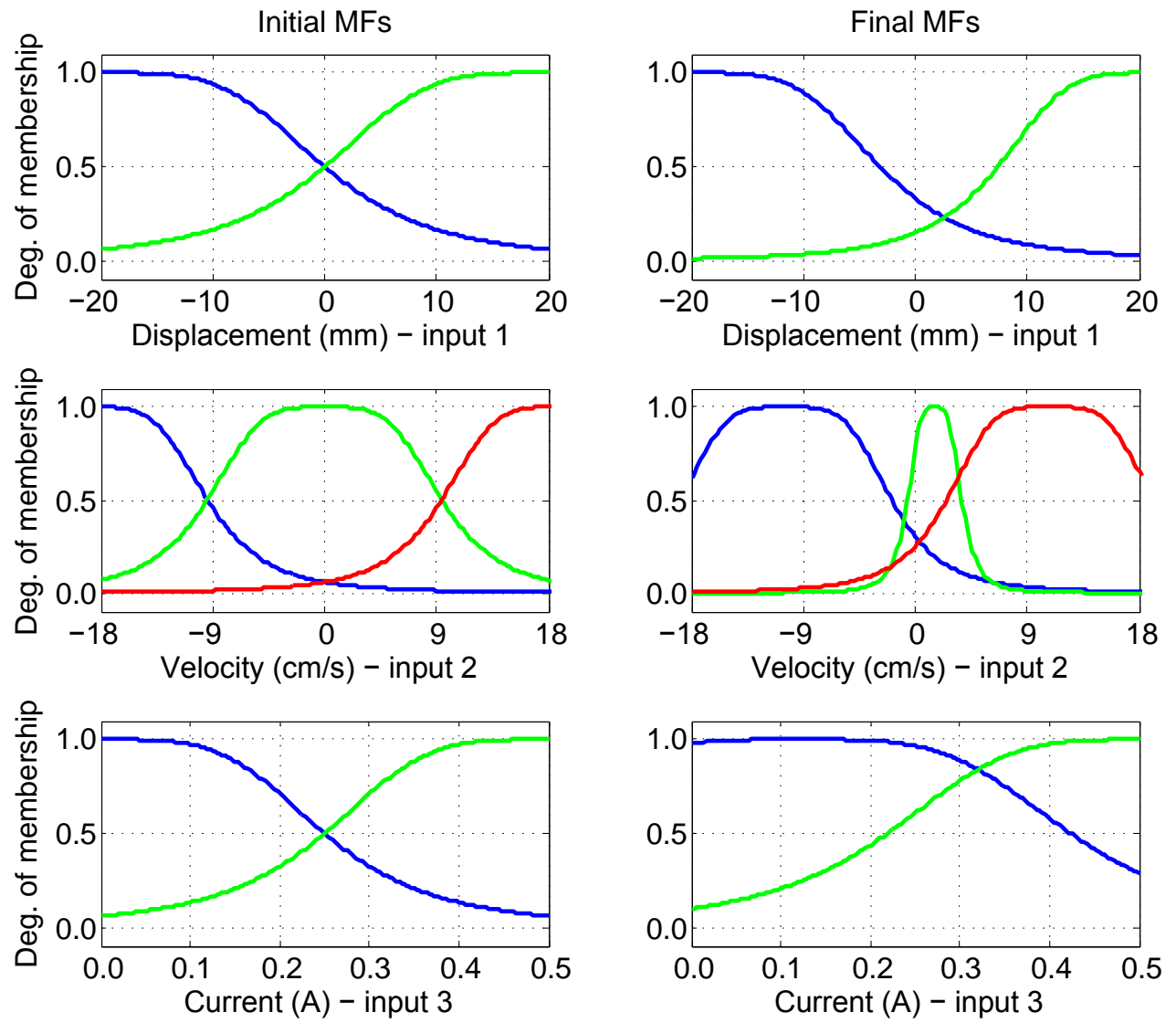

Figure 5: Input membership functions before and after training. 
The fuzzy model was obtained after an iterative trial and error process to determine the appropriate number of membership functions and the corresponding training parameters. Several training parameters (number of epochs, initial step size, error tolerance, etc.) must be defined before starting the learning process. The ANFIS training options used in the present study are presented in Table 3.

\begin{tabular}{c|c|c|c|c}
\hline Number of epochs & $\begin{array}{c}\text { Initial step } \\
\text { size }\end{array}$ & $\begin{array}{c}\text { Increasing } \\
\text { step size }\end{array}$ & $\begin{array}{c}\text { Decreasing } \\
\text { step size }\end{array}$ & $\begin{array}{c}\text { Error } \\
\text { tolerance }\end{array}$ \\
\hline 200 & $0.11 / 0.15$ & 1.20 & 0.80 & 0.1 \\
\hline
\end{tabular}

Table 3: ANFIS training parameters (RD-1097-1 MR damper).

These input MFs were obtained with an initial step size of 0.11 , which provided the best ANFIS optimization results in predicting the training data. The units of the fuzzy variables were also scaled to improve the optimization procedure (i.e., $[\mathrm{m}]$ to $[\mathrm{mm}],[\mathrm{m} / \mathrm{s}]$ to $[\mathrm{cm} / \mathrm{s}]$, respectively). In accordance with the estimated MFs, ANFIS generated a FIS structure with a total of 24 if-then rules to map the non-linear response of the MR damper. A plot of the fuzzy surface for the trained FIS model representing the output force as a function of the piston displacement and velocity, $f_{\mathrm{MR}}=f\left(\operatorname{displ}_{\mathrm{i}}, \mathrm{vel}_{\mathrm{i}}\right)$ is shown in Figure 6 .

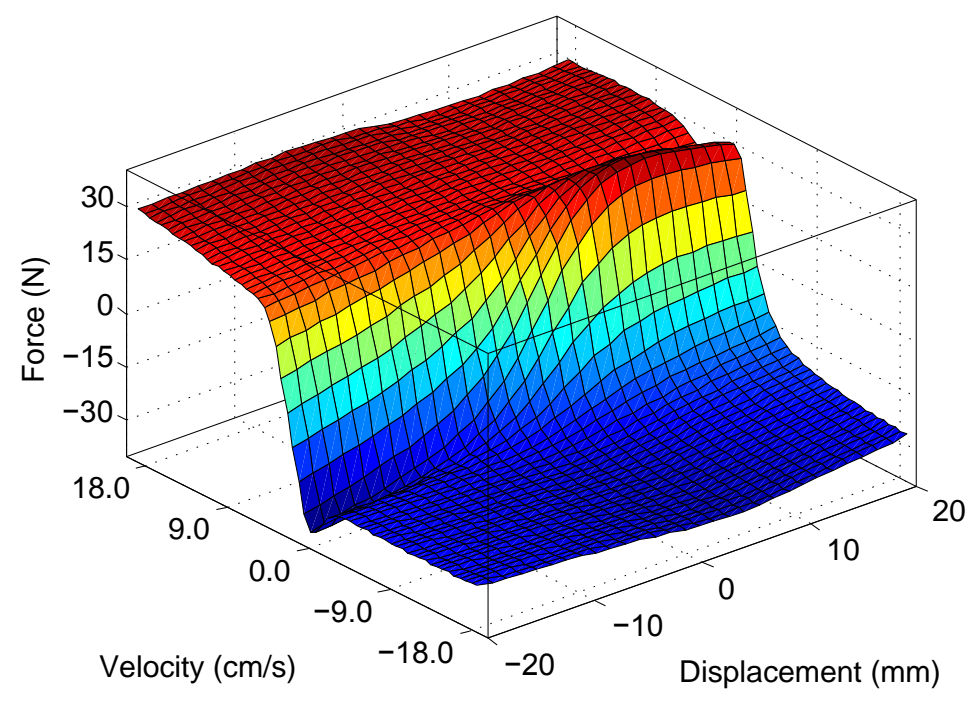

Figure 6: ANFIS estimated fuzzy surface $f_{\mathrm{MR}}=f\left(\operatorname{displ}_{\mathrm{i}}, \mathrm{vel}_{\mathrm{i}}\right)$.

The fuzzy surface has an appropriate scale with a realistic geometry in accordance with the expected MR damper behavior. Moreover, changing the velocity for small displacements generates a significant change in the damper force while changing one of these input variables when both have the same magnitude has almost no effect in the output force.

Another FIS surface displaying the resultant damping force as a function of the piston displacement and the level of operating current, i.e., $f_{\mathrm{MR}}=f\left(\mathrm{displ}_{\mathrm{i}}, \mathrm{cur}_{\mathrm{i}}\right)$ is displayed in Figure 7. The behavior depicted in the non-linear mapping of the trained fuzzy model is in line with the expected damper response for different operating current levels. 


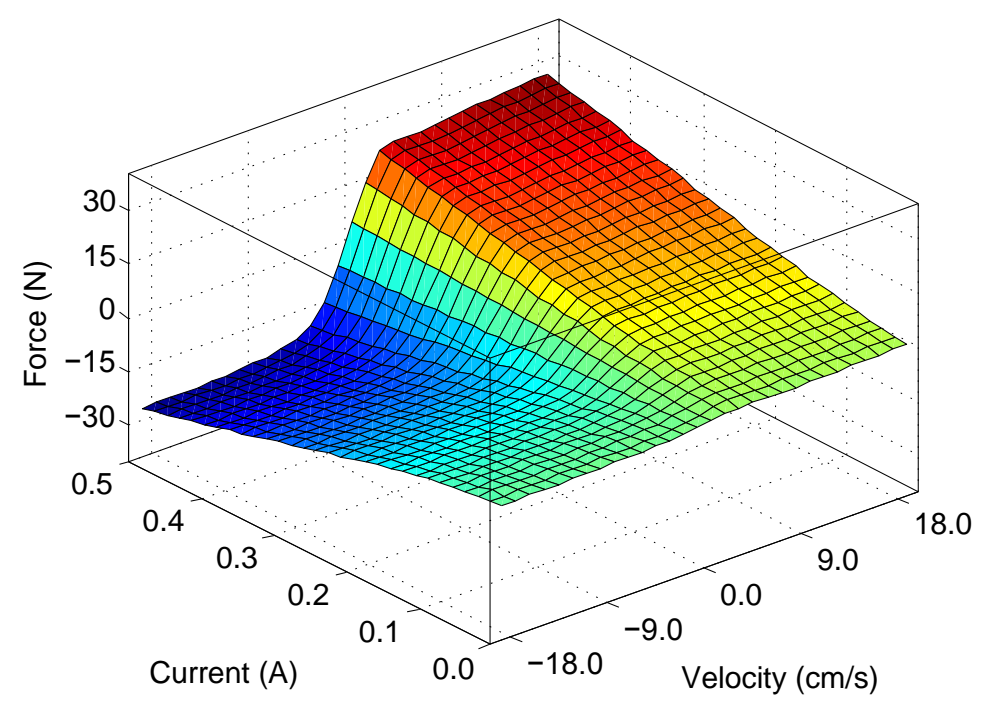

Figure 7: ANFIS estimated fuzzy surface $f_{\mathrm{MR}}=f\left(\operatorname{displ}_{\mathrm{i}}, \mathrm{cur}_{\mathrm{i}}\right)$.

The non-linear relationship between the damping force and the piston displacement with respect to different levels of operating currents is clearly visible in this fuzzy mapping, i.e., when the operating current is increased for equal values of piston displacement, the resultant damping force is also increased. Additionally, changing the magnitude of the piston displacement for low levels of command current has almost no effect in the damper force producing almost a flat surface while changing the piston displacement for high levels of operating current generates a highly non-linear force-displacement response, especially for a maximum command current where increasing the piston displacement produces a significant increase in the force developed by the MR damper.

A similar fuzzy surface representing the resultant damper force as a function of the piston velocity and the applied operating current, $f_{\mathrm{MR}}=f\left(\right.$ vel $\left._{i}, \mathrm{cur}_{\mathrm{i}}\right)$ is shown in Figure 8 . In this case, this mapping surface emphasizes the highly non-linear relationship between the damping force and the piston velocity.

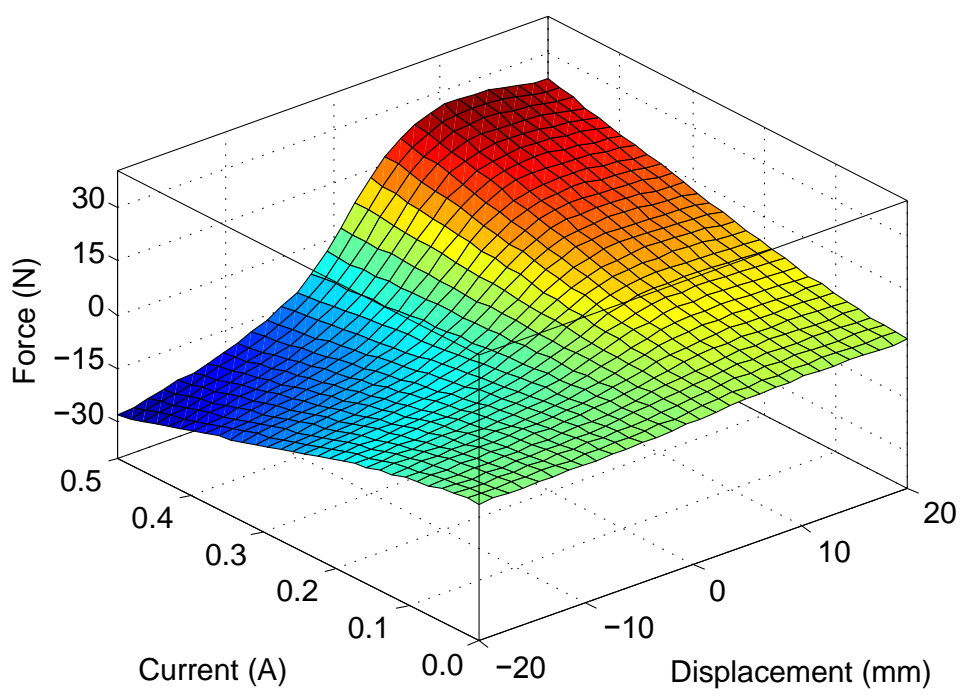

Figure 8: ANFIS estimated fuzzy surface $f_{\mathrm{MR}}=f\left(\mathrm{vel}_{\mathrm{i}}, \mathrm{cur}_{\mathrm{i}}\right)$. 
The level of damping generated by the MR damper is directly related with the damper force and the piston velocity since the gradient of the force-velocity relationship within the fuzzy surface represent the damping level. Due to the typical rheological behavior of this type of controllable devices, the damping level is a function of the current input, i.e., low operating currents generates a low damping state characterized by a flat surface while high command currents produce a high damping state represented by a steep slope in the fuzzy surface. So, increasing the operating current level will increase the resultant damper force and therefore the damping capacity of the MR damper.

The performance of the neuro-fuzzy model can be assessed by examining the convergence trend of the RMS error and step size evolution for the training and checking data over the number of epochs. Figure 9 shows the error plots along with the corresponding step size evolution. As can be seen, there is no model overfitting during the training procedure because the checking data RMSE exhibit nearly the same evolution as the training data error. A steady RMSE is achieved after 60 epochs after which the error curves tend to stabilize without a significant RMSE and step size modification.
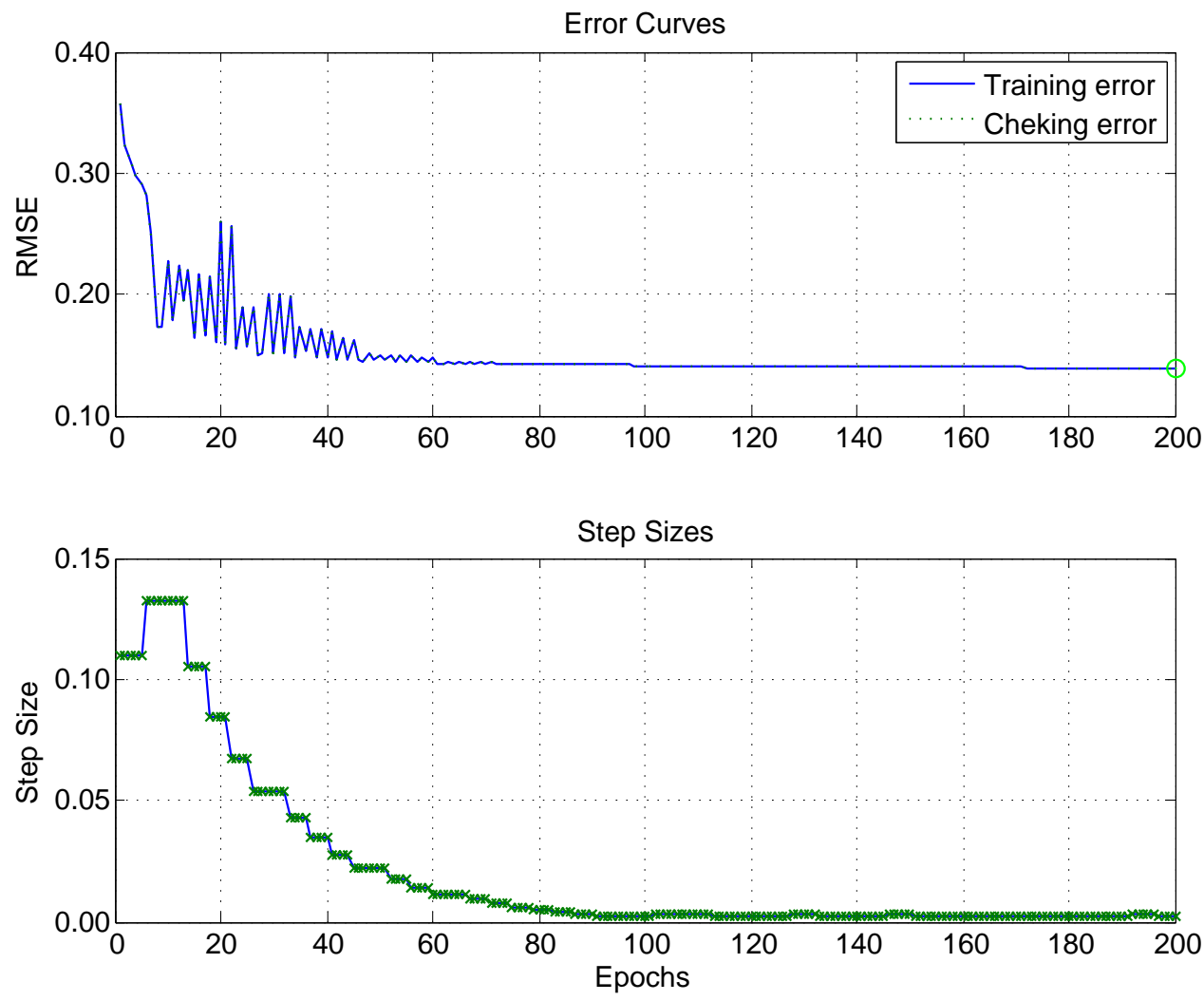

Figure 9: Training and checking errors obtained by ANFIS.

A displacement signal generated by low-pass filtering a white noise signal through a $4^{\text {th }}$ order Butterworth filter with a cutoff frequency of $4.0 \mathrm{~Hz}$ was used to generate the testing data set for the fuzzy model (Figure 10). Therefore, a testing data with $20 \mathrm{~mm}$ of maximum peak-to-peak displacement amplitude was obtained. The corresponding velocity data set was computed from displacement data using a $4^{\text {th }}$-order backward difference method. The command current was generated using a PRBS signal to represent a bi-state command signal constrained between $0.0 \mathrm{~A}$ to $0.5 \mathrm{~A}$ (maximum operating current for intermittent operation of the RD-1097-1 MR damper). 
A time step of 0.001 seconds is used to produce a total of 20000 data samples over a 20 seconds numerical simulation. The damper force was also determined from the Bouc-Wen model of MR damper. The testing data and the reference damper force are shown in Figure 10. The results achieved with the fuzzy model were then compared to those found with the reference model for the second validation data set as shown in Figure 11. There is a general good agreement between the damper force estimated with the fuzzy model and the reference data.
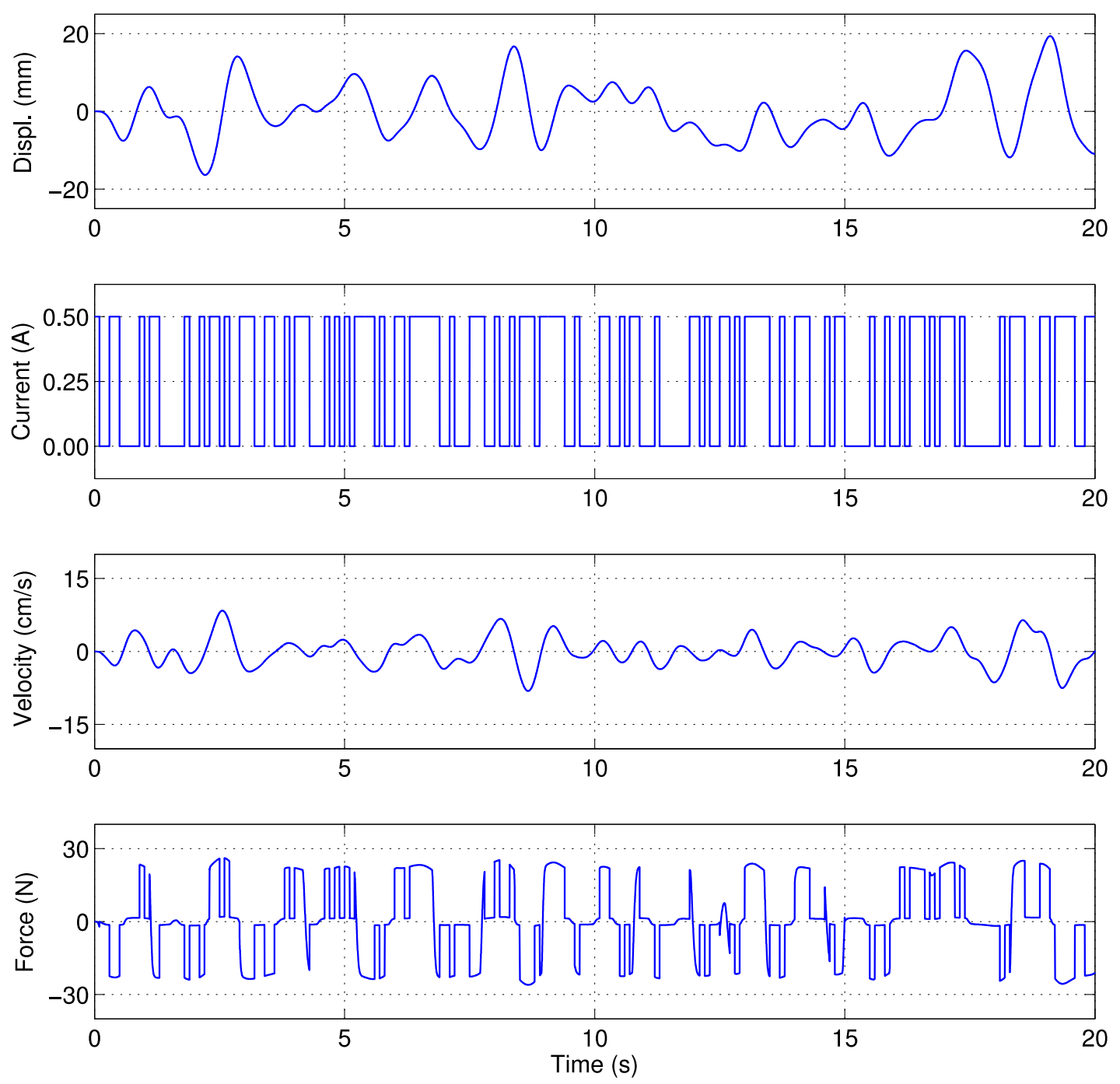

Figure 10: Testing data set for validation of the proposed fuzzy model.

An important aspect in modeling the response of a MR damper is the capability of the numerical model to represent the damper force upon a step change in the load current. It was found that the proposed model is able to represent the transient response during a command current change although the transition from pre-yield to post-yield behavior is not so accurately described. Besides peak damper forces can be slightly under or overestimated although the difference between the reference damping force and the fuzzy output force is small $\left(\mid f_{\text {ref }}\right.$ $\left.f_{\text {fuzzy }}\right|_{\max }=0.7 \mathrm{~N}$ ) resulting in a residual error below $3 \%$ relatively to the maximum damping force. In conclusion, the results indicate that the proposed neuro-fuzzy model is capable to represent with acceptable accuracy the damper response which in this case was defined using a set of artificial data from a parametric model (i.e., the Bouc-Wen model). 

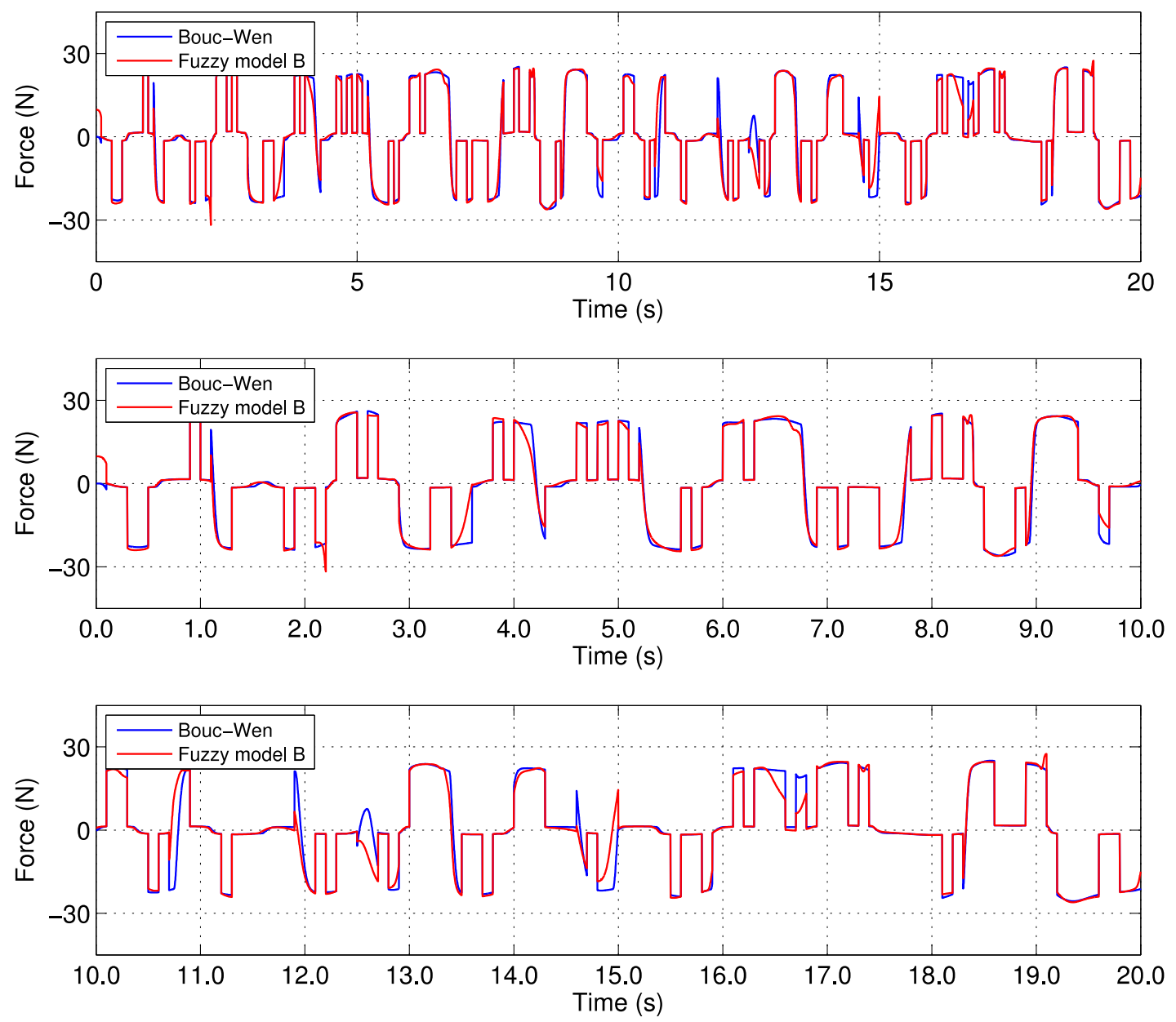

Figure 11: Results obtained with the testing data set.

The last performance assessment will be achieved comparing the experimental results achieved with a sinusoidal excitation and constant current $(5 \mathrm{~mm}$ amplitude with a frequency of $1.00 \mathrm{~Hz}$ and $0.50 \mathrm{~A}$ ) with the numerical responses obtained with both numerical models, i.e., the estimated fuzzy model and the Bouc-Wen model. The experimental and both numerical responses are displayed in Figure 12. It can be seen that, on the whole, the neuro-fuzzy model estimates the corresponding force-time, force-displacement and force-velocity experimental responses with acceptable reliability and precision being effective in capturing the size and shape of the non-linear hysteresis loop.

The already discussed mismatching between the fuzzy model prediction and the damper response around the yield point region is highlighted in the force-velocity plot (force overshoot). Although this problem affects the accuracy of the model in simulating the damper response, it does not prejudge in a significant way the final outcome by comparison with other numerical models such as the Bouc-Wen model, given that the global behavior of the MR damper can be reliably estimated. Additionally, the results obtained with the fuzzy simulation show a very good agreement with the numerical response obtained with the Bouc-Wen model. Disregarding this slight error around the yield point region, the proposed fuzzy model should be considered adequate to simulate the MR damper behavior since it provides an acceptable prediction of the device response defined by an equivalent Bouc-Wen model or the corresponding experimental data. 

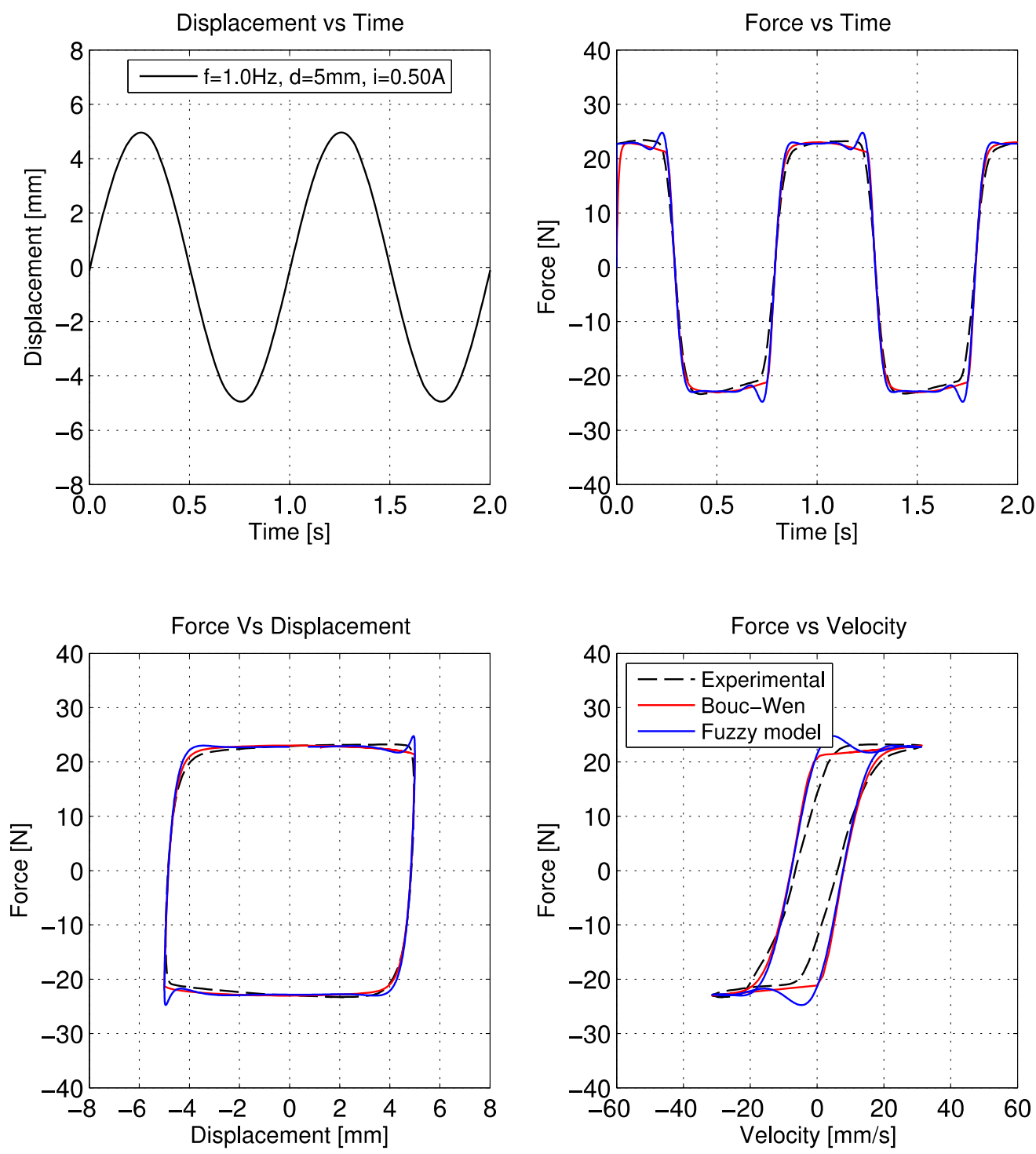

Figure 12: RD-1097-1 MR damper - Fuzzy model vs. Bouc-Wen model response $(1.00 \mathrm{~Hz}, 5 \mathrm{~mm}$ amplitude and $0.50 \mathrm{~A})$.

\section{CONCLUSIONS}

The present paper addressed a non-parametric modeling procedure for MR dampers based on an Adaptive Neuro-Fuzzy Inference System (ANFIS) model. This modeling approach was used to develop a neuro-fuzzy model for a sponge-type MR damper based on artificial training data. It was verified that the fuzzy model presents a similar numerical performance as the equivalent parametric model and therefore the proposed model is considered capable to simulate the hysteretic behavior of the MR damper. Hence, the damper response can be estimated with a relative high level of accuracy by the proposed fuzzy model. The greater the accuracy and usually the complexity of the numerical model, the better the performance in simulating the damper response. The proposed neuro-fuzzy model presents advantages and limitations that should be taken into consideration when planning and implementing a numerical model for a specific engineering application. 


\section{REFERENCES}

[1] K.C. Schurter, P.N. Roschke, Neuro-fuzzy control of structures using acceleration feedback, Smart Materials and Structures, 10, 770-779, 2001.

[2] H.S. Kim, P.N. Roschke, P.Y. Lin, C.H. Loh, Neuro-fuzzy model of hybrid semi-active base isolation system with FPS bearings and a MR damper, Engineering Structures, 28(7), 947-958, 2006.

[3] D.A. Shook, Control of a benchmark structure using GA-optimized fuzzy logic control, MSc Thesis, 2006.

[4] H. Wang, H. Hu, The Neuro-Fuzzy Identification of MR Damper, Sixth International Conference on Fuzzy Systems and Knowledge Discovery, 2009.

[5] H. Wang, Modeling of Magnetorheological Damper Using Neuro-Fuzzy System, Fuzzy Information and Engineering Volume 2, Advances in Intelligent and Soft Computing Volume 62, 1157-1164, 2009.

[6] L. Zong, X. Gong, C. Guo, S. Xuan, Inverse neuro-fuzzy MR damper model and its application in vibration control of vehicle suspension system, Vehicle System Dynamics: International Journal of Vehicle Mechanics and Mobility, 50:7, 1025-1041, 2011.

[7] J.R. Jang, ANFIS: Adaptive-network-based fuzzy inference system, IEEE Transactions on Systems, Man and Cybernetics, 23(3), 665-685, 1993.

[8] A.R. Sadeghian, Nonlinear Neuro-Fuzzy Prediction: Methodology, Design and Applications Fuzzy Systems, The 10th IEEE International Conference on Volume 2, 2-5, 10221026, Vol 3, 2001.

[9] B.F. Spencer Jr., S.J. Dyke, M.K. Sain, J.D. Carlson, Phenomenological model of a magnetorheological damper, Journal of Engineering Mechanics, 123, 230-238, 1997.

[10] D.H. Wang, W.H. Liao, Magnetorheological fluid dampers: a review of parametric modelling, Smart Materials and Structures, 20, 023001, 2011.

[11] M. Braz-César, R. Barros, Properties and Numerical Modeling of MR Dampers, $15^{\text {th }}$ ICEM - $15^{\text {th }}$ International Conference on Experimental Mechanics, 2012.

[12] The Mathworks, Inc. Optimization Toolbox User's Guide, USA, 2012. 